\title{
L'éthique de la trilogie de Yasmina Khadra Ecrivain engagé
}

\author{
By \\ Dr. Souad Ameur Mohamed \\ Professeur adjoint en Littérature Comparée
}

Journal Of The Faculty Of Education-Mansoura University

No. 111- July. 2020 


\title{
L'éthique de la trilogie de Yasmina Khadra Ecrivain engagé
}

\author{
Dr. Souad Ameur Mohamed
}

Professeur adjoint en Littérature Comparée

ياسمينة هو الاسم المستعار للكاتب الجزائري محدد مولسهول. ولد بتاريخ ، ابنابر/كانون الأول

1900 بالقنادسة في ولاية بشار الجزائرية .خلال فترة عمله في الجيش قام بإصدار روايات أثارت الرأي العام و تتاولت قضايا ساخنة تنز أفكار الغربيين كان فيها رافضا للجنون الذي بكتسح كل مكان في العالم ، معارضا لكل من تذر ع بالدبن وخلف ور اءه حمامات من الدم.

هذا المقال عبارة عن دراسة مقارنة لثلاثية ياسمبنة خضرا المتكونة من :

Les sirènes de Bagdad

Les hirondelles de Khaboul

L'Attentat

$$
\begin{aligned}
& \text { حيث تتناول أحداث هذه الروايات ثلاث مناطف ساخنة في العالم فلسطبن، أفغانستان و العراق كما } \\
& \text { أن موضوعها الرئيس كان الإرهاب و التطرف بمختلف أنواعه. الجديد في الأهر أن باسمسنة خضرا لم } \\
& \text { بر و لو لمرة عن وجهة نظره في الموضوع بل اكتفى بطرح مختلف وجهات نظــر عبـر أبطــال و } \\
& \text { شخصبات الرواية و تبقى وجهة نظر القارئ في الأخبر هي الأهم . } \\
& \text { حاول الكاتب في ثلاثيته سنونو كابول، حوربات بغداد، و الهجوم الانتحاري إزالة سوء الفهم بين } \\
& \text { الثرق والغرب التي انشأت من خلال حدود وهمية أنشاها الرجال. أن هذه ثلاثية ليبت مهة فقط من } \\
& \text { الناحية المواضيعبة ولكن أبيضًا من الناحبة الثكلية والجمالية. فهو ببتعبر خصائص المأســاة وروايــة } \\
& \text { القصص و بقوم بذمجها بانسجام مع روايته في تصور حديث للكتابة. }
\end{aligned}
$$

\section{Résumé}

Les romans de la trilogie de Yasmina Khadra, Les hirondelles de Kaboul, les sirènes de Bagdad et L'Attentat, nous permettent d'appréhender les tenants et les aboutissants d'un mode de fonctionnement contemporain dans différents pays connus du monde entier à travers les réalités du terrorisme, de la guerre, de l'autoritarisme d'état entraînant la souffrance des populations.

La réussite de l'auteur qui est mondiale tient à la riche et à la précise description de la vie de gens ordinaires, hommes et femmes, en couples ou en familles pris dans le tumulte 
d'un arrière-plan politique qui met en exergue l'incompréhension notoire existant entre l'Orient et l'Occident.

Tous les personnages, hommes et femmes dont la psychologie est particulièrement fouillée, sont présentés sans a priori dans le souci de mettre le lecteur en situation de se faire son propre jugement sur l'ensemble de ces problèmes. Abstract

Mots-clés : terrorisme, Orient, Occident, incompréhension, relation homme-femme.

Yasmina Khadra's books of his trilogy, Les hirondelles de Kaboul, Les Sirènes de Bagdad, L'attentat, are to be read so as to understand the ins and outs of a contemporaneous functioning in different countries worldly known through the realities of terrorism, war, state authoritarianism implying the suffering of populations.

The immediate success of the trilogy comes from the very rich and precise description of ordinary men and women's lives, in couples or in families, caught in the turmoil of a political background revealing the notorious misunderstanding existing between the Orient and the Occident.

All the characters, men and women alike, in their very detailed psychological analysis are presented without apriori so as to make the reader judge by himself all those problematic situations.

Key-words : Orient, Occident, misunderstanding, relation man-woman,terrorism

\section{I- Introduction}

Le monde d'hier et d'aujourd'hui a connu l'Irak, l'Afghanistan, la Côte d'Ivoire, Israël, la Palestine, la Tchétchénie, à travers les réalités de la guerre, du terrorisme, des souffrances des populations, donnant l'image d'un monde déstabilisé. Que nous le voulions ou non, la mondialisation est un fait. Nous sommes tous connectés.

Le monde s'ouvre sur un chapitre de violence avec ses conséquences de terrorisme qui a donné naissance à plusieurs écrits relatant ces états de fait. La trilogie de Yasmina Khadra est une écriture du monde rebelle capable de nous enrichir en nous dotant d'éléments essentiels pour bien connaître le monde actuel et comprendre la diversité de ses conflits à savoir l'Irak, l'Afghanistan et la Palestine .

Le conflit israélo-palestinien-arabe, les Kamikazes, la religion, la quête de la vérité, la femme et l'amour sont tous des thèmes qui ont été abordés à maintes reprises et par plusieurs écrivains. L'œuvre de l'écrivain algérien Yasmina Khadra porte précisément sur le terrorisme qui représente aujourd'hui un problème d'une grande ampleur et Khadra tente d'en comprendre les raisons pour enfin pouvoir saisir ses impacts sur l'équilibre mondial. Dans ces trois récits on voit se manifester des préoccupations morales, sociales et idéologiques. A ce stade là on peut se demander quel est le rôle de la littérature et plus précisément le rôle de Khadra. Pourra-t-ilêtre neutre devant ce genre de problème? Ses récits peuvent être considérés 
comme outil pédagogique pour tous ceux qui sont confrontés aux questions les plus controversées.

La trilogie de Yasmina Khadra soit Les Hirondelles de Kaboul sur l'Afghanistan, l'Attentat sur Israël, et Les Sirènes de Bagdad sur l'Irak regroupe tous ces thèmes, tout en se focalisant sur la relation conflictuelle qui s'est agrandie entre l'Orient et l'Occident.

\section{Corpus d'étude:}

Les trois romans, objets d'étude se passent dans trois lieux différents. Le premier, Les sirènes de Bagdad se déroule en Irak, alors que le deuxième Hirondelles de Kaboul se situe en Afghanistan tandis que L'attentat se passe en Palestine. La trilogie de Yasmina Khadra s'inscrit dans une chronologie à trois niveaux; le premier, est paru en France dans les années 60. L'écrivain y traite des questions sociales, des injustices et des inégalités dans la société. Le récit raconte l'histoire de deux couples afghans sous le régime des Talibans d'où deux femmes s'expriment ; Mussarat la femme malade et Zunaira la brillante Avocate avec deux époux : l'un geôlier qui perdra la raison et l'autre un milicien qui perdra la vie. Deux femmes sous l'anonymat portant la burka; exemples de femmes, en plein désespoir et en colère. Des survivantes sur la terre des talibans, des êtres inférieurs vivant dans la peur et l'isolement. Ce texte parle de la condition de la femme arabe en général, et de l'afghane en particulier avec la peur et la folie qui les gangrènent alors qu'elles cherchent un moyen d'évasion.

"L'attentat» quant à lui a été publié en 2005, il porte sur l'intégrisme musulman et ses dérives terroristes. Le lecteur se trouve entrainé dans un récit au cœur du conflit israélo-palestinien. Le terrorisme touche l'humanité toute entière, l'attentat se présente comme une remise en question de la violence et des valeurs occidentales. Khadra présente ce sujet à l'aide de son personnage central, Amine un chirurgien israélien qui sauve des vies et qui refuse de prendre parti dans le conflit israélo-palestinien et qui s'arrête figé incapable de comprendre la décision de son épouse Siham qui a mis fin à sa vie en terme de Kamikaze au bon milieu d'un restaurant bondé de Tel-Aviv faisant ainsi de très nombreuses victimes, un choix volontaire pour lutter en faveur de son pays d'origine; Amine ,un mari déçu incapable de réaliser ce qui se passe, se rend à Janin où il a été frappé par une explosion et meurt à son tour de ses blessures.

«Les sirènes de Bagdad» considéré comme le troisième roman de la trilogie de Yasmina Khadra a été publié en 2006 chez la même maison d'édition Julliard. La scène de ce récit se passe dans un petit village de la province irakienne Kafr Karam. Le point de repère de cette histoire est un 
jeune étudiant, un bédouin qui s'est trouvé ravagé par un sentiment de vengeance à cause des comportements abusifs de la part des soldats américains vis-à-vis des citoyens. Ce jeune homme a été manipulé par des islamistes qui ont dû profiter de la rage immense qui le démangeait, pour le recruter. Lors de sa préparation pour sa mission, il rencontra Jalal un intellectuel arabe qui le dissuada d'aller au bout de sa mission suicidaire à Londres.

Tout un monde concentrationnaire est inhérent à toute idéologie totalitaire qui entend forger une société nouvelle ou assainir l'ancienne. L'auteur parle d'un cauchemar récurrent qui est celui du terrorisme et de la guerre. L'auteur traite des sujets très sensibles qui ont sollicité un silence de peur d'être agressé ou même assassiné. Le silence s'impose dans les familles, d'autres auraient envie de parler, tout en évitant d'imposer le réel qui provoque le malaise. Certains se refugiaient dans l'écriture tel est le cas de notre écrivain qui a pu franchir la barrière de l'interdit pour faire transmettre une image humaniste de ce phénomène diabolisé qui a presque envahi le monde entier, en évoquant le discours extrémiste dans ses trois ouvrages.

A travers ses trois récits, Yasmina Khadra a peut-être joué un rôle thérapeutique, c'est pour cette raison que ses romans ont trouvé un succès mondial.

\section{II-Un écrivain débordant les normes \\ -Ecrivain engagé}

Yasmina Khadra a été longtemps ignoré par le monde de la littérature. La notoriété de Khadra ne s'est effectuée qu'à partir des années 60. C'était un romancier intarissable. Le besoin de témoigner et de dire était pour lui un besoin urgent. Sa technique puise dans la tragédie et le conte. Il écrivait dans l'urgence. Après 36 ans de service dans l'armée, il décida de prendre sa retraite. Il fut très influencé par son père, un enfant obéissant qui n'a pas su dire non au moment où il était très attiré par l'écriture ; il a exaucé le vœu de son père durant 36 ans.

Ecrire dans le monde de khadra est synonyme de risques et de danger de mort. Il s'est obstiné à aborder des sujets durs, chauds, et extrêmes. Il parle de l'interdit et attaque des thèmes épineux capables de mettre un terme à sa vie, à savoir le terrorisme intégriste: le conflit israélo-palestinien, le conflit Irako-Américain et celui d'Afghanistan." La trilogie de Yasmina Khadra, Les hirondelles de Kaboul, L'attentat et Les sirènes de Bagdad, constitue une œuvre-limite dans la mesure où l'auteur y traite un sujet très sensible, à savoir le phénomène de l'hyper-terrorisme. Lequel sujet n'est pas 
seulement difficile à traiter, mais il fait planer sur l'auteur un risque de mort. Ecrire devient dans ces conditions synonyme du franchissement de la barrière de l'interdit. Les textes essayent de nous faire voir le terroriste, cet être diabolisé, sous un regard humain. Il n'est responsable de ses actes que peu puisqu'il est la proie d'un destin implacable."(1)

Khadra n'appartient pas à un pays où la liberté d'expression est admise. Au contraire, il s'est sacrifié en se mettant à la place de ceux qui ont peur de s'exprimer, il savait ce qu'il risquait en posant des questions cruciales comme celles de la mémoire, de la valeur et de l'authenticité des témoignages. Khadra est une personne qui a dépassé le stade de la parole et s'est engagé dans l'écrit. Sa parole est plus consistante. Ses récits sont dangereux et loin d'être innocents. Rien n'est jamais gratuit. Sa trilogie a connu un grand succès car elle est innovante. C'est un écrivain des limites, une personne d'exception et un novateur, c'est-à-dire qui risque sa vie. Il a pu traiter le malentendu entre l'Orient et l'Occident, crée par les préjugés de l'un et de l'autre, il a pu franchir les frontières imaginaires des Hommes. Il n'a pas cherché à trancher contre un camp en faveur de l'autre. Le discours de Khadra est celui d'un humaniste au talent extraordinaire. Ses paroles visent l'humanité tout entière, ses mots sont adressés à chaque personne pacifique qui dit non à la violence, non à l'anéantissement de l'Afghanistan, de l'Irak, non à la torture d'un peuple choyé de Dieu .Il n'est ni du côté de l'Occident ni du côté de l'Orient : " Car l'unique combat en quoi je crois et qui mériterait vraiment que l'on saigne pour lui est celui du chirurgien que je suis et qui consiste à réinventer la vie là où la mort $\mathrm{a}$ choisi d'opérer" (2)

Ce genre de roman qui raconte la réalité et les situations du monde a été inventé par Truman Capote, Norman Mailer, Balzac, Chateaubriand, Tom Wolf et autres; Khadra à son tour a écrit des textes comprenant des réalités concrètes et a décrit des guerres dans des pays qui existent. Il a su traduire et sentir ses frères et sœurs et ceux qui leur ressemblent:

" Il a su capter, voire fasciner un si large auditoire, c'est parce qu'il ne s'est jamais départi de sa condition de héraut d'une cause, d'un peuple, d'une

\section{${ }^{(1)}$-Référence électronique}

Youssef Abouali, «La trilogie de Yasmina Khadra. Une ouvre-limite», Sciences humaines combinées [En ligne], $5 \mid 2010$, mis en ligne le 11 décembre 2017, consulté le 14 janvier 2020. URL : http://preo.u-bourgogne.fr/shc/ index.php?id=171

(2) -Attentat, p.226 
partie de l'humanité. Il le fait avec un talent encore non égalé. C'est l'arabe qui ne vocifère pas, c'est l'Algérien lourd d'une des plus cruelles étapes dans la vie de son peuple dans le monde arabe . C'est le musulman qu'habitent les lumières d'une religion qui construit; c'est l'alchimie de tout cela qui lui a sans doute permis de forcer le discours qu'est le sien, un, un discours d'un humaniste debout ${ }^{\prime \prime(3)}$

\section{III- Titrologie}

L'importance du titre dans la trilogie rappelle que le récit évolue en fonction de ce dernier. Les Sirènes de Baghdâd avec L'Irak et tout ce qui est en relation avec ce pays, Les Hirondelles de Khaboul avec tout ce qui se passe de sociopolitique en Afghanistan et L' Attentat avec tout ce que porte ce mot de terreur de terrorisme et de mort, peu importe le lieu où il pourra se manifester. Les titres de ces romans ne sont donc pas fortuits et ce sont des présupposés, pour les personnages, de leurs destinées et de leurs identités héroïques, et pour les œuvres, de leurs contenus.

"Le titre est une inscription en tête d'un livre, d'un chapitre pour indiquer le contenu, le qualifier et le déterminer" (4)

Khadra a choisi minutieusement les titres de ses productions littéraires dans le but de les préciser et de les distinguer, rien qu'en lisant ces derniers, on voit clairement qu'il s'agit de lui, les mêmes propos et les mêmes principes. La titrologie est une discipline qui s'est intéressée à l'interprétation des titres des œuvres et à sa réception vis- à - vis des lecteurs. La valeur du titre est grandiose, rien qu'en l'interprétant, on peut facilement comprendre l'œuvre :

"Le titre est un message codé en situation de marché : il est le résultat de la rencontre d'un énoncé romanesque et d'un énoncé publicitaire en lui se croisent nécessairement littéralité et socialité : il parle de l'œuvre non pas en terme de discours social mais le discours social en terme de romans" ${ }^{\text {"(5) }}$

Cependant il ne faut pas oublier que l'interprétation d'un titre demande deux compétences, celle du lecteur et celle de l'auteur. C'est le premier élément du roman fourni par l'auteur au lecteur, la recherche du sens du titre à ce moment-là demande une participation active de la part du lecteur pour construire et donner un sens au texte:

(3)-Alwatan.com, Littérature-Yasmina Khadra dans sa trilogie; Zouaoui Benhamadi, 14 octobre 2006.

(4) - Larousse, Dictionnaire Français, France, Juin 2000, p.423. 2

(5) - G.Genette, cité par C.Achouret S Rezzoug, Introduction à la lecture du littéraire en convergence critique, Alger; OPU, 1995,P 28. 
" Le titre exerce l'aspect autoritaire entre le texte et l'écrivain qui provoque chez le lecteur une certaine méthode pour le choix de ses lectures qui est qualifié d'une structure à la fois signifiante et symbolique" (6)

Les titres présentent aux lecteurs des relations de réciprocité avec le roman qu'il identifie, les deux titres des deux premiers romans sont des syntagmes présentés avec des séries d'éléments. Ils sont constitués d'un déterminant (les), d'un nom propre (Kaboul, Bagdad) qui commencent par des articles définis, ce qui nous pousse à penser que c'est une information connue, une situation politique, socio-historique bien définie. L'Attentat, le troisième titre est formé d'un syntagme nominal, un déterminant défini (le) et suivi d'un nom "Attentat" qui dit bien des choses, parce que, qui dit "Attentat" dit trahison, dit terrorisme, dit peur, mort, cadavre, orphelins. Rien que ce nom, nous donne un aspect péjoratif des choses. L'image qui accompagne le titre symbolise le contenu du texte; c'est un signe fort qui interpelle automatiquement le lecteur puisqu'il lui transmet une information précise.

Kaboul et Bagdad sont deux noms propres de villes en plein chaos :"Kaboul est devenue l'antichambre de l'au-delà. Une antichambre obscure où les repères sont falsifiés; un calvaire pudibond; une insoutenable latence observée dans la plus stricte intimité"(7)

Symbole de conflits" Bagdad est l'endroit le plus dangereux de la terre aujourd'hui" ${ }^{(8)}$ Deux villes portant le signe d'une guerre entre deux peuples, deux civilisations; arabe et occidentale : "Nous sommes en plein choc de civilisations. Il va te falloir choisir ton camp." ${ }^{(9)}$ Alors que le titre Attentat ne marque pas de lieux ; mais rien qu'en lisant le titre, on se rend compte qu'il s'agit d'un acte suicidaire, on se retrouve face au grand conflit Arabo- Israélien. L'auteur met le lecteur en garde. C'est vrai que rien n'est mentionné concernant une ville ou un pays, mais on comprend dès les premières pages qu'il s'agit de la Palestine et du grand conflit Israélo-arabe : "Deux peuples élus qui ont choisi de faire de la terre bénie de Dieu un champ d'horreur et de colère. Je ne me souviens pas d'avoir applaudi le

(6) - Asma Marir, Dans L'Enjeu De L'Intertextualité/Dialogisme Etude Onomastique Et Comparative Du Privilège Du Phénix De Yasmina Khadra Et de L'As De Tahar Ouettar, mémoire en science des textes littéraire, Ecole Doctorale de Français Antenne de l'Université Kasdi Merbah- Ouargla,2008-2009, P 18.

(7)- Les sirènes de Baghdâd, Yassmina Khadra, Edition Julliard, 200 6,P .12

(8) -Les sirènes, p.50

(9) -Les sirènes, P.292. 
combat des uns ou condamné celui des autres, leur trouvant à tous une attitude déraisonnable et navrante. Jamais je ne me suis senti impliqué, de quelque manière que ce soit, dans le conflit sanglant qui ne fait, en vérité, qu'opposer à huis clos les souffre-douleur aux boucs émissaires d'une histoires scélérate toujours prêtes à récidiver" ${ }^{\prime 10)}$

Le nom "Attentat" dans le contexte de la guerre palestinienne est porteur de plusieurs significations, il désigne Chahid comme il désigne Kamikaze, Mojahid, fidayeen il est assimilé à l'éternité et à Jannàa et alors qu'il est assimilé pour les occidents à terrorisme; mort; intégrisme, diable. Deux peuples à mentalités différentes. Khadra attire l'attention du lecteur vers des lieux de l'histoire. De là, on remarque qu'il présente ses romans à travers l'actualité et à travers l'histoire de ces trois pays.

Deux grands thèmes traversent ces récits celui de la mort et celui de l'incompréhension entre Occident et Orient. Géographiquement ce sont des lieux les plus chauds sur cette planète. Les noms de ces villes laissent apparaitre intégrisme, terrorisme et guerre. La citation de deux animaux porteurs de symboles dans le titre n'est pas le fait du hasard. L'auteur a choisi ces deux animaux avec précaution. En revenant au dictionnaire on trouve qu'hirondelle est synonyme de voyage, d'immigration comme il est symbole de séparation et de solitude, comme on peut aussi lui attribuer un autre symbole celui du changement et du renouveau, comme elle peut être symbole de peur et d'angoisse."

" Le ciel afghan, où se tissaient les plus belles idylles de la terre, se couvrit soudain de rapaces blindés: sa limpidité azurée fut zébrée de trainée de poudre et les hirondelles effarouchées se dispersèrent dans le ballet des missiles. La guerre était là. Elle venait de se trouver une patrie"(11)

Quant à la sirène c'est un animal mythique. A son tour il est porteur de symbole «Dans la mythologie grecque, les Sirènes sont des créatures ayant le visage d'une femme sur un corps de poisson. Elles vivaient sur une île (Sirenum Scopuli ; trois petites îles rocheuses) et avec le charme irrésistible de leur chanson elles entraînaient les marins vers leur destruction sur les roches entourant leur île" ${ }^{\prime 12)}$. L'hirondelle, cet oiseau a de multiples sens ; il peut être séducteur comme il peut être un monstre comme déjà cité dans les histoires mythiques, comme il peut insinuer tout simplement les

(10) -Attentat, Yassmina Khadra, Edition Julliard, 2005, P.163

(11)-Attentat,. p14.

(12) - Tristan Mandon "Les Origines de l'Arbre de Mai" dans la cosmogonie runique des Atlantes boréens http://racines.traditions.free.fr 
sirènes des ambulances transportant les blessés et les morts dans un pays en pleins chaos, comme il peut s'agir aussi de Ferouz la fameuse chanteuse libanaise "Aucun ange ne lui arriverait à la cheville. Ce n'est pas une sirène qui chante, c'est le souffle cosmique qui se délecte de son éternité"(13)

Comme elle peut être aussi le chant que le héros composait " Je l'ai intitulé Les sirènes de Bagdad"(14) suite au choc et à l'humiliation que les forces de l'ordre lui ont fait subir lors de leur intervention à l'improviste chez lui après la mort subite de son cousin Soulayman " Les souvenirs de la bavure me tourmentait sans relâche. Dès que je m'endormais, les cris du GI noir me sautaient dessus. de Souleyman en train de courir, l'échine roide, les bras ballants, le corps penché tantôt d'un côté, tantôt de l'autre. Une multitude de minuscules geysers giclait dans son dos. Au moment où sa tête explosait, je me réveillais en hurlant. Bahia se tenait à mon chevet, une casserole pleine de compresses gorgées d'eau"(15)

Les trois romans abordent l'histoire de trois grandes villes à travers les traits des personnages qu'ils présentent. Ces titres donnent une idée sur le contenu de chaque roman, le sens, l'interprétation par la suite dépendra de chaque lecteur:

" - Je l'ai intitulé Les Sirènes de Bagdad.

-Celles qui chantent ou bien celles des ambulances?

-C'est à chacun de voir.

Les titres de cette trilogie sont chargés de connotations et ont un rapport étroit avec l'histoire du roman: « Le roman traduit son titre, le sature, le décode et l'efface où le réinscrit dans la pluralité d'un texte et brouille le code publicitaire en accentuant la fonction poétique latente du titre, transformant l'information et le signe en valeur, l'énoncé dénotatif en foyer connotatif $\gg{ }^{(16)}$.

On peut remarquer aussi que les titres de ces deux romans portent des noms féminins, ce qui renforcera la forte présence du féminin dans les trois récits objets d'étude. Sur les deux couvertures se trouvent aussi des photos de femmes portant le tchador, ce qui insinue la présence à travers les signes du roman de la condition socio politique de la femme dans un état en pleine guerre. Le grand sourire de la femme-enfant sur la couverture du roman Sirène de Bagdad est signe de l'innocence signe aussi d'une

(13) - Les sirènes de Baghdâd, Yassmina Khadra, Edition Julliard,2006,P93

${ }^{(14)}$-Les sirènes, p.82

(15) -Ibid, p78

(16) -Gérard genette, Les titres, Coll., Poétique, Seuil, 1987, p. 76 
"Génération Espoir". L'espoir est une disposition de l'esprit humain qui se repose sur l'idée de l'attente d'une situation meilleure à celle de l'existent. Une des émotions, qui donne envie de vivre et donne l'envie de s'engager encore plus pour trouver remède à tous les problèmes sur terre.

Espoir et le signe du mythe de Pandore, cette femme dans la mythologie grecque qui a su refermer la jarre juste à temps pour y conserver l'espoir de l'humanité toute entière. Cette mythologie raconte comment les dieux se sont vengés des mortels en leur envoyant la première femme, Pandore, qui a ouvert le couvercle de la jarre où sont enfermés tous les maux de la terre, c'est ainsi qu'ils se sont échappés pour accabler l'humanité.

"Mais Bagdad était un passoir, Elle Prenait l'eau de partout. Les attentats y étaient monnaie courante.... Un stand tir, une gigantesque boucherie.....Partout, à travers la planète à Rome comme à Tokyo, à Madrid, et à Paris, au Caire et à Berlin, les peuples défilaient en masse- des millions d'inconnus convergeaient sur le centre de leurs villes pour dire non à la guerre. Qui les avaient entendus ?. La boite de Pandore ouverte, la bête immonde se surpassaient. Plus rien ne semblaient en mesure de l'assagir."

L'auteur parle de Pandore dans son récit ce qui renforce mon interprétation des choses et le fait qu'il $\mathrm{y}$ a espoir dans les générations à venir, que le dialogue sur lequel insiste l'écrivain peut être une solution grâce aux générations qui suivent surtout que sur la couverture de Attentat, on retrouve encore une fois l'image d'un enfant en train de voir ce que font les adultes. La photo dans Attentat pourra véhiculer deux connotations, celle de l'espoir déjà cité auparavant et une celle d'une autre connotation qui est celle d'une génération qui pourra dissoudre cet espoir et l'anéantir à jamais dans une ultime tentative. Une génération qui suivra les mêmes pas celle qui l'a précédée, tel est le cas dans la construction de l'histoire qui débute et s'achève par la même scène. L'attentat est encadré par deux chapitres similaires comme si l'histoire était écrite à l'envers et la structure du temps dans ce roman est circulaire. La même scène qui ouvre et ferme l'Attentat peut ainsi dire que l'être humain et les générations tournent dans un cercle vicieux et que le conflit entre l'orient et l'occident est perpétuel et continuera à travers les générations qui suivent et ne prendra pas fin. Les attentats et les ripostes soit en Palestine, en Irak ou En Afghanistan ou ailleurs ne règleront rien, ce n'est pas une résolution de conflit, il est urgent de chercher un atmosphère de dialogue et d'entente et il est temps que nos enfants ne nous montrent plus du doigt et tâchent de changer cette réalité que l'écrivain cite dans Sirènes de Bagdad en disant : "L'occident ne nous 
aime pas. Il ne t'aimera pas, toi non plus, il ne te portera pas en son cœur puisqu'il n'en a pas et ne te portera jamais aux nues puisqu'il te prend de haut, tu veux rester un minable lèche-bottes, un Arabe servile un raton privilégié; tu veux continuer d'espérer d'eux ce qu'ils sont incapables de te donner? OK. Tu prends ton mal en patience et tu attends. Qui sait? Un nonos pourra tomber de leur sac-poubelle."(17)

La Page titre avec tout ce qu'elle porte, elle est révélatrice et provoque la curiosité du lecteur, les photos sont des éléments complémentaires qui invitent à la lecture ; le créateur du titre transmet un message chargé de signification, le lecteur le déchiffre. Le roman et le titre comme l'auteur et le lecteur sont tous les deux complémentaires et donnent sens au roman, le roman traduit le titre le décode et lui donne plus de valeur, c'est un référent très important.

\section{IV-Etude des personnages dans la trilogie de Khadra}

a-: présentation des personnages féminins du roman.

Khadra veut donner un élément de vrai par la présentation des fragments de l'histoire contemporaine. Dans ce cas le narrateur partage avec ses personnages le même point de vue et perçoit la même vision avec eux. Il réside au-dedans du personnage. Les textes ne présentent pas le point de vue du narrateur parce qu'il ne reflète pas sa subjectivité comme dans l'autobiographie. Khadra essaie de comprendre, de trouver des vérités et de chercher au-delà de cette réalité de misère, de malheur, d'ignorance, le terrorisme et l'injustice. Il n'est pas un simple être, il voit les gens de manière différente et les traite différemment. Il se trouve obligé de réfléchir à toutes ces notions. Nous pouvons dire que les personnages ont une focalisation zéro. Cette dernière sert à bien décrire les personnages de l'intérieur en expliquant tous leurs ressorts psychologiques et concentre l'étude sur le fond de la personne. Au contraire, en focalisation interne, le narrateur reste fidèle au mystère de la vie en respectant l'opacité des êtres et l'étrangeté de leurs mobiles. Ces mots sont parfaitement chargés de sens, de valeurs et de perceptions idéologiques puisque l'auteur essaye de montrer l'utilité du nom en rapport avec l'idéologie : il se détermine dans le roman par le choix sélectif et opératif d'idées qui établissent des influences performatives chez le lecteur surtout occidentaux, ils sont amalgamés avec des personnes vraies dans l'histoire qui ont existé et qui existent dans la

(17) -Les sirènes, p. 290 
mémoire collective d'un peuple en entier. Comme Saddam Houssain, Cheik Yacin, Rafik Hariri, Bush, Areal Charon.

La structure de l'individu comme être vivant oblige et exige l'étude du personnage sur les trois plans en parallèle : physique, moral et social. Les personnages dans les trois récits dictent des idées, ce sont des clichés et des modèles exceptionnels. Khadra a enrichi la texture de son roman par l'intégration des idées et des points de vue variés qui peuvent justifier ou donner des explications au thème de la résistance, de la révolution, du refus et de la rupture avec Israël. Tous les personnages de la trilogie excepté le héros, occupent une place secondaire dans le roman. Sans eux l'intrigue serait tout à fait différente et la cohérence du récit serait quasi impossible, le héros a besoin d'autres personnages qui vont l'épauler. Ces personnages secondaires vont soutenir son rôle de héros, pas nécessairement être de son côté pour bien mener sa quête. Mais, ils peuvent, en effet, être des ennemis, des amis, des membres de la famille, etc. Ils sont là pour donner la réplique au héros, pour équilibrer les traits qui le caractérisent. Cependant, certains de ces personnages secondaires sont du premier plan, participent au déroulement des événements et sans eux l'histoire aurait pris un autre tournant. Par ailleurs, il existe un autre type de personnages secondaires. Il s'agit des personnages d'arrière-plan, qui n'apparaissent dans le roman que de manière ponctuelle. Ils sont liés à un lieu ou à une situation vécue par le héros et qui disparaissent dans la suite du récit. Ces personnages que croise le héros et qui peuplent le roman contribuent à l'animation de l'espace romanesque.

Le nom, le portrait physique, la psychologie, la biographie (âge, état civil, hérédité biologique et sociale, un passé, etc.) sont tous des indices très importants pour étudier les personnages dans les trois récits. L'être du personnage est la somme de ses propriétés à savoir son portrait physique et les diverses qualités que lui prête le romancier. Il conçoit l'être du personnage comme « le résultat d'un faire passer » ou « un état permettant un faire ultérieur ». Donc, son être est difficilement séparable des autres aspects du personnage: de son faire, de son dire, ou de son rapport aux lois morales.

Le nom propre donné au personnage est un élément important pour l'individualisation de tout personnage car il s'agira du nom d'une seule personne bien précise ; c'est un instrument de "l'effet du réel », alors que l'auteur donne des noms à chaque protagoniste dans les deux premiers romans il s'abstient de le faire pour le héros du 3ème roman, il s'est contenté de lui donner un référent de bédouin, un personnage typique, plus 
proche de lui, un personnage qui incarne toutes les valeurs arabes. L'absence risque de déstabiliser le lecteur car comme le signale David Lodge :« dans un roman les noms ne sont jamais neutres, ils signifient toujours quelque chose... Nommer un personnage est toujours une étape importante de sa création. " En plus du nom donné au personnage, l'auteur le caractérise par le corps, la psychologie et la biographie. De plus, de qui s'agit-il ? Le personnage chez Khadra incarne différentes fonctions, parmi ces rôles, il peut véhiculer des valeurs morales tel est le cas avec le personnage secondaire d'Adel dans L'Attentat, il a presque pu grâce à ses propos convaincre Amine à propos de l'acte suicidaire de son épouse :" Je bute contre la transparence d'une vitre, je vois clairement son message, mais impossible d'y accéder"(18), lorsqu'il lui dit :"Sihem ne voulait pas de ce bonheur-là. Elle le vivait comme un cas de conscience. La seule manière de s'en disculper était de rejoindre les rangs de la Cause. C'est un cheminement naturel quand on est issue d'un peuple en souffrance. Il n'y a pas de bonheur sans dignité, et aucun rêve n'est possible sans liberté. Le fait d'être femme ne disqualifie pas la militante, ne l'exempte pas. L'homme a inventé la guerre, la femme a inventé la résistance. Sihem était fille d'un peuple qui résiste. Elle était mieux placée pour savoir ce qu'elle veut" ${ }^{\prime 19)}$

Je vais commencer par présenter les personnages-femmes de Khadra dans les trois récits objets d'étude même si elles sont minoritaires et ne sont pas présentées comme les héroïnes des récits car leur présence dans le déroulement des faits est très importante. Le nombre de fois où la protagoniste Sihem, sujet de discussion est citée, est considérable, elle est citée en majorité par les autres personnages du roman; masculins et féminins. "Sihem était une sainte. Un ange"(20) . Elle était belle jeune et forte. Durant tout le récit on parle d'elle, et c'est grâce aux autres personnages qu'on est suffisamment informés d'elle puisqu'elle est morte avant même le début du récit. Elle représente le personnage typique de la femme arabo-musulmane qui ne s'exprime pas beaucoup, mais qui passe tout simplement à l'acte et qui s'affirme dès que le moment convenable arrive. Sihem se manifeste dès les premiers instants de l'intrigue. A travers ce personnage, Khadra nous montre les efforts accomplis par des femmes pour être reconnues comme personne à part entière dans la société, et non plus comme un être soumis, sous tutelle d'un père ou d'un époux comme

\footnotetext{
(18)-Attentat,p.220

${ }^{(19)}$-p. 220

(20)-Attentat, p.216
} 
l'était Missurat, un être entièrement soumis à son mari, mais qui a passé à l' acte au moment approprié ; elle a pris l'initiative et a mis fin à sa vie de son plein gré en se substituant à Zuneira lors de son exécution. Zuneira dans Hirondelles de Kaboul est la fille d'un notable, une belle femme " plus captivante que jamais" (21) elle a connu son mari à l'université. Ce personnage constitue l'espoir d'une société; elle est la seule qui s'est opposée au règne des talibans et a manifesté son refus, elle est le signe de toutes les femmes qui aspirent à la liberté et à l'égalité.

Faten est un personnage secondaire très peu développé par l'auteur. Cette femme est citée une seule fois dans le récit, c'est un personnage secondaire mais, elle occupe toutefois une place importante dans le roman. C'est à cause d'elle qu'Amine s'est dirigée vers Janin, là où il est mort par un drone .Il est allé la chercher, après qu'elle a disparu de la maison patriarcale enlevée par les soldats israéliens comme réplique de l'attentat suicidaire que son frère avait perpétré. Amine savait au fond de lui qu' il y aurait une revanche à prendre. Farah, quant à elle, est rêveuse et très ambitieuse, et ce grâce à l'instruction solide qu'elle a reçue à l'université, alors que l'éducation de la femme était inhabituelle à cette époque. L'éducation avait changé sa vie, elle avait un regard sur le monde différent de celui des autres femmes afghanes."C'était une rebelle et une battante, la seule fille de Kafr Karam, à avoir osé enfreindre les règles tribales et faire exactement ce qu'elle avait envie de faire .Son audace et son insolence avaient certainement forgé son tempérament en la rendant plus agressive et moins conciliante." ${ }^{(22)}$

Zuneira et Missurat les deux personnages de Hirondelle de Kaboul ainsi que les autres personnages femmes de la trilogie n'avaient pas le droit de choisir ni le droit de se déplacer sans être accompagnées parfois même de leur mari; elles se retrouvent dans des situations d'humiliation comme dans la scène qui a déclenché le passage à l'acte pour Zuneira dans les Hirondelles de Kaboul ". Elle a toujours milité pour la cause féminine, avec l'arrivée des Talibans, elle s'est trouvée humiliée, dépourvue de la moindre liberté même lorsqu'elle est accompagnée de son mari: "Ton épouse va t'attendre ici, au pied de ce mur, en retrait. Tu la raccompagneras plus tard" (23)

\footnotetext{
${ }^{(21)}$-p. 57

(22) - Les sirènes de Bagdad, p. 149

(23) - HK ,p. 71
} 
Les qualifications que l'auteur a attribuées à Zuneira, Missutat, et à l'héroïne Sihem dans Attentat ainsi qu'au personnage secondaire Faten sont loin d'être fortuites ; ces qualifications révèlent les ambitions et les rêves de femmes de s'imposer dans la société en tant que femme et de choisir, même si c'est le choix de mourir. Zuneira a détesté son mari et a décidé de le repousser à jamais et de l'expulser de sa vie; suite à une querelle familiale, Zuneira s'est trouvée condamnée à mort à cause de son acte qui a valu la mort de ce dernier. Elle y a échappé par miracle grâce au geôlier qui l'aimait ardemment et à son épouse Misurata qui s'est substituée à elle lors de l'exécution sans qu'elle ne s'en rende compte. Ces protagonistes ont un pouvoir primordial sur le cours des événements essentiels dans la trilogie. Ce n'est pas uniquement les autres protagonistes qui font d'elles des héroïnes, mais principalement, leurs quêtes qui ont fait d'elles des personnages présents dans les romans et des jeunes femmes hors du commun dans la société.

Kadra nous présente des femmes hors du commun dans la société arabo-musulmane en pleine guerre. Elles représentent espoir et révolte sous le joug d'une société patriarcale. A un moment donné dans le récit Zuneira dit " avec ce voile maudit, je ne suis ni un être humain, ni une bête, juste un affront ou une opprobre que l'on doit cacher telle une infirmité" (24)

Ainsi, nous pouvons dire que Zunaira, cette femme heureuse et très ambitieuse et ce grâce à l'instruction solide qu'elle a reçue à l'université alors que l'éducation de la femme était inhabituelle sous le règne des talibans, avait un regard sur le monde différent de celui des autres femmes afghanes; elle s'est obstinée à s'imposer dans la société en tant que femme faible, privée de toute liberté ; Néanmoins, leur impact sur l'homme est fort même si elles se trouvent isolées; c'est pour cette raison que ces femmes sont présentées par khadra comme actrices sociales malgré leurs conditions difficiles de femmes. Sihem dans Attentat était comme la plupart des femmes :"Elle était si bien dans sa cage dorée, n'est-ce pas? Elle mangeait bien, dormait bien s'amusait bien. Elle ne manquait de rien." ${ }^{\prime(25)}$ Cependant et malgré tout elle a décidé de prendre parti pour la Cause palestinienne, elle a sombré dans le militarisme suicidaire :"Personne n'y est pour quelque chose. Je ne voulais pas qu'elle aille se faire exploser, mais elle était déterminée .Même l'Imam Marwan n'a pas réussi à l'en dissuader. Elle a dit

\footnotetext{
(24)-hk ,p. 71

(25) - attentat, p.206
} 
qu'elle était palestinienne à part entière et qu'elle ne voyait pas pourquoi elle laisserait d'autre faire ce qu'elle devait faire" (26)

Sihem même morte est restée le pivot du récit tout entier, l'héroïne sujet. La quête de son mari a changé après qu'elle a décidé de commettre un acte suicidaire. Sa mort a causé un grand désordre dans la vie d'Amine.

Il ne faut pas envisager le sens de héros ici, comme simplement personnage principal. Certes Zuneira occupe une place importante dans le roman, c'est un personnage féminin rebelle au sein d'une société où la femme est soumise et marginalisée. Elle se distingue des autres personnages féminins du roman par ses agissements extraordinaires, ses mérites exceptionnels et ses exploits dignes des grands héros. "...elle est sublime, d'une fraicheur inaltérable .Malgré les inclémences quotidiennes et le deuil d'une ville livrée aux hantises et à la folie des hommes" .C'est elle qui a poussé le geôlier à changer son ordinaire et à faire un acte que lui-même est incapable d'expliquer.

Le couple de Zuneira a perdu sa famille, son commerce, sa maison, elle et son mari ne mangent plus à leur faim, mais ils sont restés ensemble, ils se sont soutenus pour nourrir leur espoir, Mohsen aimait son épouse à la folie "Tu es le seul soleil qui me reste, Zuneira sans toi, ma nuit serait plus profonde que les ténèbres, plus froide que les tombes." Les personnages de Khadra dans les trois romans vacillent entre réalité et fiction. Ce thème se manifeste dès les premiers instants de la lecture de l'intrigue. A travers ces personnages, nous constatons que ces femmes font tout pour être reconnues comme personne à part entière dans la société, et non plus comme un être soumis, sous tutelle d'un père ou d'un époux ou d'une société intégriste; tel est le cas de Zuneira et Missurat qui n'avaient pas le droit ni de choisir ni de se déplacer. Les qualifications par exemple que l'auteur a attribué à l'héroïne Zuneira, sont loin d'être fortuites ; ces qualifications révèlent les ambitions et les rêves de ce personnage d'avoir les mêmes droits que l'homme, de s'imposer dans la société en tant que femme moderne et de se débarrasser des traditions. Pour être libre elle a transgressé les lois rigides de la société elle a fait de sa personne une femme instruite dont la voix n'était jamais sans écho comme l'était celle de toutes les femmes de l'époque à Kaboul.

De ce relevé de personnages de femmes dans le récit, on parvient à distinguer clairement deux catégories de femmes qui se diffèrent par des

(26) - attentat, p.214 
comportements et des attitudes qui les confinent à des rôles différents. Ainsi, on distingue des jeunes femmes rebelles tel Sihem, Zuneira, Faten et des femmes soumises à leurs sorts tel Missurat, . Toutes les femmes citées dans ce roman illustrent la condition de la femme et son statut au sein de la société arabo-musulmane. Avoir une instruction solide ne l'aidait pas autant mais c'était le seul moyen de s'affirmer et de se faire une place dans la société afin d'affronter les grandes injustices que les femmes subissaient. Ainsi, la société présentée par Khadra est représentée par l'ensemble de ces personnages féminins de différentes cultures et régions agissant essentiellement autour de l'absence et la présence de la femme dans la société ou dans le cas de Zuneira par le biais de l'instruction. L'instruction parfois leur permettait de passer de la soumission à la prise en main de leur destin. Tel était le cas de cette dernière et de Sihem entre autres. Ces femmes dévoilent la triste vérité de la mise en arrière-plan de la femme dans la société Afghane, Irakienne ou encore palestinienne par les traditions et les croyances et ensuite par la guerre. Cependant, les femmes sont présentées dans ce roman comme des êtres dociles qui soutiennent l'homme. Face à cette souffrance accrue des personnages féminins dans le roman, seule Sihem a su franchir le cap non parce qu'elle a accompli cet acte affreux mais parce qu'elle a pu faire ce qu'elle croit juste de faire. Cette jeune femme rebelle représente toutes les femmes palestiniennes qui tâchaient de lutter pour exister, défendre leur liberté et résister à leurs ennemis pour préserver leur pays. Khadra, à travers le personnage de Sihem et de Faten, souligne la participation de la femme dans les combats armés contre les Israéliens, ou avec Zunéira dans Les Hirondelles de Kaboul. L'écrivain souligne tous les obstacles qui entravent la femme malgré son instruction, à combattre pour leur émancipation contre le bourreau de taliban et contre les extrémistes dans le monde entier. Dans ces romans dès que les personnages apparaissent au sein de la société comme des êtres forts, on se dépêche d'effacer leur présence au nom de la morale et au nom de la religion musulmane, mais Zuneira luttera et se révoltera, c'est ce qu'on comprend à travers la fin tragique proposée par l'écrivain. Il a laissé la fin du récit ouvert avec la disparition de cette dernière, sans nous donner le moindre détail sur son sort:" Abasourdi, terrifié, le coeur sur le point de lui jaillir de la gorge, il retourne au milieu de la pelouse, exactement là où une flaque de sang s'est coagulée et, la tête dans les mains, il scrute obstinément une à une, 
les tribunes. Soudain, réalisant l'ampleur du silence, ses mollets cèdent, et il tombe à genoux. Son cri de bête foudroyée se déverse sur l'enceinte, aussi épouvantable que l'effondrement d'un titan; Zuneira." ${ }^{(27)}$

\section{b: La représentation des personnages masculins du roman.}

Le bédouin: héros du récit Sirène de Bagdad, ce personnage nous a été présenté pourvu de nom, un personnage pauvre et sensible . Il a tant cherché à réaliser son vœu de devenir docteur ès lettres alors que son projet a été totalement anéanti après l'invasion de l'Irak par les forces de la coalition. Il décida de ne pas prendre parti et se retira vers son village natal pour vivre en paix loin de tout ce qui est en rapport avec la politique et la guerre, un être pacifique. Seulement le destin et la chance étaient loin de lui procurer une telle paix, l'honneur familial a été transgressé en la personne la plus honorée, le père ! Ce qui obligea le bédouin à crier vengeance, un tel acte n'est guère acceptable : "Le soir, quand je m'allonge sur le canapé en face de la fenêtre, je me remémore les vacheries qui ont jalonné ma vie, et toutes renforcent mon engagement. J'ignore ce que je vais faire exactement, quelle sera la nature de ma mission $-\ldots$ quelque chose qui ramènera le11 septembre à un chahut de récré. Une certitude absolue: Je ne reculerai pas! Dit-il ${ }^{(28)}$

Dr. Jalall: dans Sirène de Bagdad est présenté comme un docteur universitaire, il a enseigné durant de longues années en Occident. Il a toujours été invité sur les plateaux de chaines télévisés européennes comme un analyste du fléau intégriste djihadiste. C'est une personne qui a réussi dans sa vie comme académicien professionnel, il a été une personne armée de savoir et de connaissances étant alors le pire ennemi des extrémistes, seulement il a compris qu'il ne valait rien pour les Occidentaux et qu'il n'était qu'une arme pour combattre les siens, ce n'est qu'à ce moment-là qu'il a décidé de changer de cap: "A leur yeux, je n'étais qu'un Arabe assassin du cinéaste. Ils avaient radicalement changé, eux, les précurseurs de la modernité, les plus tolérants, les plus émancipés des Européens. Les voici qui arboraient leur tendance raciste comme un trophée. Pour eux, désormais, tous les Arabes sont des terroristes, et moi?...Moi, Djalal, ennemi juré des fondamentalistes, moi qui croulais sous les fatwas, qui me cassais le cul et les dents pour eux? Moi à leurs yeux, je n'étais qu'un traitre à ma nation, ce qui me rendait doublement méprisable... Et là, j'ai eu comme une

(27) $-\mathrm{HK}, \mathrm{p} .141$

(28) - Attentat, p. 258 
illumination. J'ai compris à quel point j'étais dupe, et surtout, où était ma vraie place. J'ai donc plié bagage et j'ai rejoint les miens." ${ }^{(29)}$

Soupçonné d'être un traître, il parcourt le pays arabe et musulman pour prêter son talent et son intelligence redoutable aux directives djihadistes. Il est d'un franc parler qui dépasse l'ordinaire. Il a surpris sa mère avec un autre homme alors qu'il était encore enfant et il en parle et fait des aveux et étend son linge sale comme le dit le bédouin alors que de telles révélations dans un village arabe- musulman sont mortelles. Il a essayé auparavant de se suicider à cause de l'acte déshonorant de sa mère .C'est un homme solitaire, il a toujours peur d'être assassiné et la solitude lui pèse. Ce personnage intellectuel a apporté plusieurs explications au malentendu qui existe entre l'occident et l'Orient ; ses paroles apportent des éclaircissements aux différentes notions qui demandent des explications, c'est bien lui qui a comparé l'Occident à un chant de sirènes pour les naufragés identitaires .Ce n'est qu'un mirage; là encore, on retrouve une autre connotation au mot Sirène pour dire que l'occident, comme les sirènes mythiques, n'apporte avec leurs chansons mensongères que le malheur et la mort. Ce n'est que $\mathrm{du}$ mirage, tout est mensonge : "Parce qu'ils adhéraient aux valeurs occidentales, ils prenaient pour agent comptant ce qu'on leur susurrait à l'oreille: liberté d'expression, droit de l'homme, égalité, justice...des mots grands et creux comme les horizons perdus. Mais tout ce qui brille n'est pas or.......Pourtant, ça crevait les yeux qu'ils se battaient pour des prunes. Jamais leurs confrères occidentaux n'allaient leurs permettre d'accéder à la reconnaissance. Le vrai racisme a toujours été intellectuel. La ségrégation commence dès lors qu'un de nos livre est ouvert." ${ }^{\prime(30)}$ Ce personnage est un acteur dialogique et idéologique. Dr Jallal et le Bédouin sont deux personnages tout à fait différents, ils ne sont pas de la même pâte et leurs foix sont différentes.

Mohsen et Atiq sont les deux héros dans Hirondelles de Kaboul, Mohsen est un homme instruit, qui a connu son épouse à l'université et a toujours soutenu la cause féminine ; objecteur de conscience, jamais rien ne l'a convaincu à prendre des armes ou donner la mort. Une cravache toujours à la main, il a cessé de rêver, sa conscience s'est éteinte, presque chaque jours il assiste à des exécutions " La mort pour lui et pour les autres n'est qu'une banalité. D'ailleurs, tout est banalité. Hormis les exécutions qui

(29) - SB, p. 264
(30) - SK, p. 17 
réconfortent les survivants chaque fois que les mollahs balaient devant leur porte ." Son père était négociant prospère, "son bonheur était plein" avec l'invasion soviétique à l'âge de 10 ans tout a changé" ${ }^{(31)}$ Après être humilié par les Talibans alors qu'il était accompagné de son épouse, Mohsen a changé il se sent devenir fou. L'impact de cette humiliation était très profond. Jamais ce jeune homme ne pourra accepter d'être l'ennemi de qui que ce soit, jusqu'au jour où il a participé à la lapidation d'une prostituée, sans en prendre vraiment conscience, il a participé avec la foule qui a réclamé le sang, il a été choqué par son geste " Je n'arrête pas de regarder ces mains que je ne reconnais plus" ${ }^{(32)}$ Il s'est confié à sa belle épouse qui s'est trouvée tétanisée par le récit de son mari et a pris un comportement radical. Elle s'est éloignée de lui "Mohsen comprend qu'il n'aurait pas dû confier à sa femme ce que lui-même refuse d'admettre" ${ }^{(33)}$ Pour aller en avant en guise de réconciliation ils se sont décidés à sortir en promenade et comment a-t-il pu croire, comme le cite le narrateur dans le texte, que les promenades d'amoureux étaient encore possibles dans une ville aux allures de mouroir, infestée d'énergumènes rébarbatifs portant dans le regard la noirceur de la nuit des temps? Et Comment a-t-il pu perdre de vue les horreurs qui jalonnent le quotidien d'une nation bafouée au point que la cravache est devenue une langue officielle?

Atiq Shaukat est le geôlier dans les prisons des femmes de Kaboul et un ancien moujahid qui a participé à la guerre contre l'union soviétique. Il est devenu fou à la fin du récit après avoir perdu les deux femmes de sa vie. Un homme âgé de 42 ans, avec une barbe d'intégriste, des yeux soulignés de khôl perdant toute acuité: "ne voit ni le bout du tunnel ni celui de son propre nez" un homme déjà usé avec une âme blessée qui a perdu toute confiance, il a connu son épouse, l'infirmière, lorsqu'il s'est blessé, son travail lui a joué un mauvais tour, il s'est trouvé au bon milieu d'un malaise perpétuel, la maladie incurable de son épouse ainsi que la mort qu'il voit tous les jours l'ont poussé vers l'extrême, il est devenu une personne triste, errant, cherchant l'amour, la vie et le bonheur partout ; une fois qu'il rencontre Zuneira, il tombe amoureux, ne voyant que cet amour et oubliant tous les principes reçus comme étant un Taliban : "Il ne se sent pas près de rentrer chez lui retrouver son lit défait, la vaisselle oubliée dans les bassines

\footnotetext{
(31) - Hirondelles de Kaboul p, 12

(32) - P31

(33) $-\mathrm{p} 32$
} 
malodorantes et sa femme couchée en chien de fusil dans son coin, la tête ceinte dans un foulard crasseux et la figure violacée"(34)

Amine, un être pacifique, naturalisé israélien. Le personnage principal dans l'Attentat est une construction compliquée qui frappe notre attention et notre conscience par les affinités de sens qu'il traduit. Il refuse tout propos et ne cherche qu'une seule réponse qui est celle de la cause qui a pu pousser son épouse au suicide kamikase .Il est fier d'être Palestinien et fier de son appartenance, il revendique son état et assume ses responsabilités envers l'humanité comme étant médecin "...llan Ros m'a profondément affecté . Pourtant, je n'affiche ma religiosité nulle part. Depuis l'université, j'essaie de m'acquitter scrupuleusement de mes taches citoyennes .Conscient des stéréotypes qui m'exposent sur la place publique, je m'évertue à les surmonter un à un, offrant le meilleur de moi-même et prenant sur moi les incartades de mes camarades juifs" ${ }^{\text {"(35) }}$

Le récit dans Attentat est présenté à la première personne par un narrateur qui relate des événements passés alors qu'il est mort. Sa participation à l'intrigue est en fait présentée sous la quête identitaire en tant que produit de l'énonciation, où sa prise de parole dialogique constitue une interrogation purement idéologique dans le roman.

Une valeur idéologique est véhiculée dans le récit par les personnages soit principaux ou secondaires .Leurs paroles demandent toujours à être lues. Les personnages sont bien choisis dans les textes de Khadra. Ils assurent la lisibilité du discours et orientent vers des réponses aux questions du jour: " La liberté n'est pas un passeport que délivre la préfecture, partir où l'on veut n'est pas liberté. Manger à sa fin n'est pas la réussite. La liberté est une conviction profonde : elle est mère de toutes les certitudes" ${ }^{\prime(36)}$

Amine, Atiq, le Bedouin et Dr Jallal sont des points centraux dans les trois récits, ils réalisent le rôle du narrateur dans le roman. Ils rapportent, décrivent et commentent ce que le romancier veut présenter et affirmer aux lecteurs. Les personnages exposent des idées, des remarques qui peuvent rapprocher l'image de la vérité aux lecteurs afin d'arriver à une bonne compréhension des faits soit En Irak, à Kaboul ou en Palestine. "Virus ou bombe, qu'est-ce que ça change, lorsqu'on étreint d'une main une offense et de l'autre la Cause? Je ne prendrai pas de comprimé pour dormir. J'ai réintégré mon élément. Tout va bien. La vie n'est qu'un pari insensé;

(34)- Les sirènes de Baghdâd, Yassmina Khadra, Edition Julliard, 2006, p.35

(35) - Attentat, p. 99

(36)-Attentat, p.219 
c'est la façon de mourir qui lui sauve la mise .Ainsi naissent le légendes"(37) Le discours dans les trois récits est purement idéologique à travers ce dernier se dégage une image philosophique de la personne. " Aujourd'hui, la conscience du monde, c'est nous. Toi et moi, et ces intelligences orphelines, conspuées par les leurs et dédaignés par les cerveaux encroutés. Nous sommes minoritaires mais nous existons" (38)

" Hier seulement un immense suaire la frappait de fond en comble, confisquant ses lumières et ses échos, ramenant ses excès d'autrefois à une misérable angoisse de la feuille blanche, faite de froidure er de perplexité, de grave échec et d'incertitude...A-t-elle oublié son martyre au point de ne pas compatir au deuil de ses proches ? Indécrottable, Beyrouth. Malgré le spectre de la guerre civile qui gravite autour de ses festins, elle fait comme si rien n'était ....Quelle aube, avec les lendemains ?.... Non, je ne finirai pas comme eux. Je ne veux même pas leur ressembler." ${ }^{(39)}$

Les personnages transmettent des idées ainsi que des valeurs vouées à l'humanité toute entière; Ils envoient un message universel, de relation de respect et rejettent l'injustice et la mort. " Nous avons une lourde responsabilité sur les épaules, Jalal. Tout dépend de nous, de toi et de moi. Notre victoire est le salut du monde entier. Notre défaite est le chaos. Nous avons un instrument inouï entre les mains : notre double culture .Elle nous permet de savoir de quoi il retourne où est le tort et où est la raison, où se situe la faille chez les uns et pourquoi il y a un blocage chez les autres. L'occident est dans le doute. Ses théories, qu'il imposait comme des vérités absolues, s'émiettent dans le souffle des protestations. ${ }^{(40)}$

Les positions de l'écrivain ne se manifestent que sous la pression des événements ; on ne voit guère de préjugés, mais des énoncés qui se formulent par le narrateur et par le personnage principal au fur et à mesure que les événements dans le roman progressent. Deux types d'images circulent dans le récit, on constate que Khadra regroupe deux camps sans donner raison ni à l'un ni à l'autre, il ne faisait qu'exposer les différentes opinions ; parmi les amies les plus proches d'Amine, Kim Yehuda, et Ezra Benhain des israéliens qui l'ont soutenu tout le long de son trajet alors qu'il était en train de chercher la réalité, la vérité qui a poussé son épouse à un tel acte si affreux

\footnotetext{
${ }^{(37)}$ - Les sirènes p. 280

${ }^{(38)}-\mathrm{p} .285$

${ }^{(39)}$-Les sirènes, p. 279

${ }^{(40)}$ - p 287
} 
"Je n'ai pas l'intention de me venger je veux juste comprendre comment la femme de ma vie m'a exclu de la sienne" ${ }^{\text {(14) }}$

A côté de ces israéliens, on voit la présence de Mosché, un agent de police qui a des préjugés anti-arabes :" Pour moi, tous ces fumiers se valent, qu'ils soient du Jihad islamique ou du Hamas, ce sont les bandes de dégénérés prêtes à tout pour faire parler d'elles "(42)

L'auteur présente aussi d'autres personnages, cette fois-ci palestiniennes, "les Imams extrémistes" des chefs de guerre, mais pour qui l'auteur n'a aucune sympathie et qui pousse le lecteur vers un jugement purement négatif. L'auteur et le texte rejettent leur discours et ne donnent guère raison au terrorisme. Dans les trois romans circule une certaine sagesse vis-à-vis des souffrances que subit l'humanité frappée par le terrorisme. L'écrivain décrit des souffrances sans prendre parti. Cette sagesse est articulée autour des personnages qu'Amine croise tout le long de l'histoire soit des protagonistes israéliens ou palestiniens. L'écrivain ne dénonce pas une partie et ne prend pas de position contre non plus. Il ne fait que présenter les différents points de vue de toutes parts et c'est au lecteur de voir les choses et d'en juger.

Par ailleurs, on réalise dans les trois romans objets d'études, la présence du terme humiliation, Khadra nous transmet la signification de ce terme à l'aide de son protagoniste dans Attentat en disant: " il n'est pire cataclysme que l'humiliation. C'est un malheur incommensurable, docteur. $\mathrm{Ca}$ vous ôte le gout de vivre. Et tant que vous tardez à rendre l'âme, vous n'avez qu'une idée en tête: comment finir dignement après avoir vécu misérable, aveugle et nu?" ${ }^{(43)}$

Tout acte violent a été déclenché par un acte d'humiliation. C'est le point déclencheur de toute violence dans les trois romans pour dire que partout existe l'extrémisme et que chacun de nous peut devenir à un moment donné un kamikaze. Selon Khadra l'humiliation en est la cause. Ce motif de l'affront et de l'humiliation a été pris dans les trois romans avec la scène de l'assaut de la marine américaine dans Sirènes de Bagdad lorsqu'ils ont tiré le père de famille par force de son lit en exposant aux yeux de ses enfants ses organes génitaux:" Le soleil pouvait toujours se lever, plus jamais je ne reconnaitrais le jour de la nuit... Un Occidental ne peut pas comprendre, ne peut pas soupçonner l'étendue du désastre. Pour moi, voir le
(41) - P. 288
(42) $-\mathrm{P} 42$
${ }^{(43)}$ - Les sirènes, p.212 
sexe de mon géniteur, c'était ramener mon existence entière, mes valeurs et mes scrupules, ma fierté et ma singularité à une grossière fulgurance pornographique.... les portes de l'enfer m'auraient été moins inclémentes ! [...] je sus que plus rien ne serait comme avant, [...] que, quoi qu'il advienne, j'étais condamné à laver l'affront dans le sang"(44)

L'humiliation a été vécue aussi dans Les Hirondelles de Kaboul avec la scène des Talibans après l'altercation de ces derniers dans la rue de Kaboul; ils ont interrompu la petite promenade de Zuneira avec son mari tout en l'humiliant et en le traitant avec les pires injures: "On ne rit pas dans la rue insiste le sbire. S'il vous reste un soupçon de pudeur, rentrez chez vous et enfermez-vous à double tour.... Qu'est-ce qu'il y a? Le nargue le taliban ...Tu veux me crever les yeux? Vas-y, montre voir ce que tu as dans le ventre, face de fille!"(45).

Suite à cela Mohsen ne distinguait plus le jour de la nuit et quelque chose d'irréversible avait sanctionné sa vie.

Ce même thème a été repris dans Attentat lors de la destruction de la maison ancestrale d'Amine par les forces de l'ordre israéliennes comme riposte à un attentat suicidaire. D'ailleurs un des personnages dans l'Attentat le dit clairement :" Il n'est pire cataclysme que l'humiliation. C'est un malheur incommensurable, docteur. Ça vous ôte le gout de vivre. ${ }^{(46)}$

Ces discours sont élaborés par khadra dans un but précis, ce ne sont pas des actes subis, ils ont vraisemblablement un but précis non pas pour donner raison aux actes violents mais surtout pour expliquer au lecteur occidental quelque chose de la culture arabe qui a un grand impact sur la psychologie de la personne. On remarque aussi que khadra donne la parole aux personnages pour dire et expliquer leurs points de vue extrémistes non pas pour donner raison à ses personnages qui ont touché le fond des choses, mais pour donner la possibilité au lecteur de comprendre d'où vient le mal et les mettre à distance.

On peut dire donc, que la volonté de Khadra dans sa trilogie est d'une part de rendre justice aux femmes et de rétablir leur image au sein de la société. $\mathrm{Au}$ sein de ses récits, l'Histoire est largement respectée soit irakienne, palestinienne ou afghane. Ceci d'une part, d'autre part, le genre littéraire dans lequel s'inscrit la trilogie de khadra comme ; "roman historique » impose une très forte présence masculine aux moments les plus

\footnotetext{
(44)- Les sirènes, p. 113

(45) - H.K, P. 70

(46) -Attentat, p. 212
} 
marqués de l'Histoire et de l'histoire. C'est-à-dire que l'importance actantielle attribuée aux personnages masculins est due à l'authenticité des événements historiques et des personnages. Il a su répondre à l'attente du lecteur par la présence masculine indispensable au bon fonctionnement du roman historique. Sans manquer aussi de marquer la présence féminine ancrée dans la vie et dans l'Histoire des peuples.

Le fonctionnement des personnages, masculins et féminins, dans le roman obéit finalement aux normes de vraisemblance historique. En effet, même si la plupart des romans de Khadra ont comme personnage principal un homme et que la femme se cantonne à des fonctions principales dans le récit, l'auteur ne néglige guère la frange féminine mise en marge dans la société, et on constate que son importance est indéniable car elle remplit des fonctions très symboliques dans ces romans qui remontent dans l'Histoire pour mettre en scène la condition de la femme arabe et son statut à un moment de guerre. Développer ainsi ces personnages féminins, en mettant l'accent sur leur enfermement et leur silence, permet à l'auteur d'écrire un roman qui regorge de stéréotypes culturels et de lever ainsi le voile sur la vérité de la société où les femmes ont souvent été condamnées au silence et à la soumission aux hommes. Avec L'Attentat l'écrivain nous montre que la femme pendant la guerre peut être non seulement la partenaire de l'homme, mais aussi celle qui détermine ses choix et sa destinée alors qu'elle peut être parfois mythifiée ou idéalisée et parfois stéréotypée. L'auteur nous fait savoir que les personnages féminins de ce roman vacillent entre réalité et fiction. Les femmes, malgré les efforts de l'auteur, n'arrivent pas à se détacher de la place subalterne qu'elles occupent dans la société. Le romancier nous a exposé un ensemble de personnages avec des caractéristiques différentes dans le but de spécifier et de mettre son interprétation dans le sens où le personnage romanesque se définit sur trois pôles son être, son faire et son savoir.

Le faire du personnage est présenté par des informants et des indices propres à l'être : le nom, le sexe, le milieu social, la profession, etc. La fonction de ces informants et de ces signes ne peuvent être utile qu'au moment de l'interprétation du roman parce qu'ils demandent au lecteur de les repérer et de les exploiter pour fournir et établir un sens du personnage romanesque.

\section{V-Conclusion}

Les trois récits présentent une histoire contemporaine, d'un point de vue romanesque le roman se situe entre le réel et la fiction, le réel historique et le réel romanesque, les textes représentent le troisième millénaire, les 
trois romans suivent les mêmes lignes de pensée, écrire l'histoire contemporaine sous forme d'un roman avec la sélection de trois capitales ciblées dans les guerres occidentales .Les textes parlent des rêves, à travers lesquelles les protagonistes changent la réalité de façon fantastique, la forme romanesque est échappatoire, les personnages de fictions sont amalgamés par ceux de la réalité selon la théorie qui dit que dans la fiction se réalise l'impossible qui ne se réalise pas dans la vraie vie. L'écrivain a mimé le réel à l'aide des marques explicites et directes, faciles à imaginer, pour produire les portraits des personnages. Il a donc inventé un monde semblable au nôtre par l'introduction d'un autre monde fictif et ce, afin de le rendre acceptable par rapport au lecteur par le procédé de la vraisemblance. En décrivant le personnage, l'écrivain souhaite présenter une identité très précise, où la description aide au dévoilement de l'état de la personne en donnant son portrait physique, moral et sa place dans la hiérarchie sociale. Nous réalisons aussi que Khadra a donné plus de repères référentiels dans son premier récit Sirènes de Bagdad que dans les deux autres récits. Des noms de villes et des noms des personnalités bien connus dans le monde entier se véhiculent dans le premier récit alors que seulement deux personnalités sont mentionnées dans Hirondelles de Bagdad et aucune n'est dite dans L'Attentat.

Le thème apparait dans les romans objets d'études. L'écrivain essaie de transmettre un message au lecteur, un message humaniste à tout le monde. Les romans ont aussi une approche historique. Il traite une problématique de très haute importance qui est celle de l'hyper-terrorisme pour pouvoir comprendre la vérité, l'universalité, le sens de la vie et de la nature humaine et de l'existence; un mélange. Il a cherché des vérités locales ; en effet plusieurs écrivains algériens ont subi des sorts tragiques lorsqu'ils ont traité des sujets aussi chauds. Une cascade de données nous submerge; on n'arrive plus à cerner le réel de l'imaginaire, l'auteur fait référence aux événements vrais, aux lieux qui existent évidemment, à des personnalités et à des gouvernements et institutions réels, il a reproduit la réalité. Dans Sirènes de Bagdad beaucoup d'événements se sont succédé au Liban, Kafr. Karam et Bagdad, le lieu de naissance du héros, suivis de l'invasion du pays par les soldats américains, l'histoire du héros et celle de l'Irak avec son invasion en mars 2003 et la capture de Saddam. L'auteur a parlé aussi de la chaine ELJazira dont chaque Arabe connait l'ampleur et l'influence sur les révolutions et les guerres dans le monde arabe. Cette chaîne a eu une forte portée dans le suivi du déroulement des événements en Irak. 
"Il faut bien dire que cette chaine a joué un rôle cardinal dans cette guerre. Elle a contrebalancé le poids de la chaine américaine d'information $\mathrm{CNN}$ et leur a arraché l'hégémonie qu'elle avaient sur le paysage médiatique mondial" ${ }^{(47)}$

La trilogie de Khadra a un effet de réel et elle s'inscrit dans une actualité brûlante, il a planté des repères spatio- temporels à travers le viseur tout en véhiculant le contenu du monde. Il a ensuite fait le choix d'écrire en français ce qui n'est pas fortuit de la part d'un écrivain algérien. Il dit à ce propos qu'il a opté pour la langue française parce qu'elle lui a tout appris : son histoire, le monde, les Autres, les rêves les plus fous, les peines les plus éprouvantes. C'est donc par pure gratitude qu'il la revendique. Toujours avec Khadra, on se retrouve entre réel et imaginaire, Histoire et histoire .Dans Attentat, on retrouve des noms de villes bien connues sur le plan mondial tel Bethléem, Janin, Alaqsa, aussi on se retrouve devant les événements du 11 septembre, un événement connu sue le plan mondial comme étant un Attentat d'une très grande influence. Fellouga la ville cité dans Attenta a été en réalité assiégé en avril 2002 et attaquée par l'armée israélien visant Cheikh Yacin le 22 mars 2004 et mentionnée justement par l'auteur. Dans Hirondelles de Kaboul aussi, on assiste à un autre événement dans la réalité celui de l'entrée de Taliban le 26 septembre 1996 suivi de l'invasion d'Afghanistan par les forces de la coalition dirigée par les EtatsUnis d'Amérique en octobre 2001. Tout un chapitre présenté par Khadra parle de Taliban de la charia de la loi islamique en donnant plusieurs exemples de la lapidation de la prostituée, la peine de mort pour les blasphémateurs et pour la femme qui a tué son mari. Il a parlé aussi des pratiques comme celui de l'interdiction des cerfs volant de la musique le port du tchador pour les femmes et de la barbe pour les hommes à coté de tout cela on retrouve des références du présent et de l'Histoire de ce pays comme celui des monarchies et l'âge d'or, les armés de résistance lors de l'occupation soviétique en 1979 source d'honneur et de gloire pour les Mojahidines.

La littérature maghrébine d'expression française dans laquelle s'inscrit Kahdra traduit la réalité locale, sa littérature n'est plus écrite pour amuser le lecteur, le faire rêver ou le distraire mais elle est là pour la recherche de la vérité de l'homme et du monde qui l'entourent. L'essence de

(47) -telephon 
la littérature ici est la vie ; l'auteur a un souci du réalisme et de l'imaginaire c'est un art du mélange esthétique.

\section{Bibliographie}

*Corpus

1- Yasmina Khadra, Sirène de Bagdad,

2- Yasmina Khadra ,Hirondelles de Kaboul,

3- Yasmina Khadra, L'Attentat,

\section{* Euvres}

1- Youssef AbouAli, Yassmina Khadra ou la recherche de la vérité, Etude de la trilogie sur le malentendu entre l'Orient et l'Occident, l'Harmattan, 2013.

2-De l'utopie totalitaire aux œuvres de Yasmina Khadra, approches des violences intégristes, Louisa khadra, Harmattan, 2007.

3-L'écrivain, Une enfance algérienne, Yassmina Khadra ,Poket, Paris, 2001.

4-Poétique du politique dans l'œuvre de Yasmina Khadra, Mohamed Boudjadja, Paris, 2009.

5-Poétiques de la violence et récits francophones contemporains, Emmanuel Bruno Jean-François, Livre électronique, Leiden Bostone Brill Rodopi , 2017.

6-G.Genette, cité par C.Achouret S Rezzoug,Introduction à la lecture du littéraire en convergence critique, Alger; OPU, 1995.

7-Asma Marir, Dans L'Enjeu De L'Intertextualité/Dialogisme Etude Onomastique Et Comparative Du Privilège Du Phénix De Yasmina Khadra Et de L'As De Tahar Ouettar, mémoire en science des textes littéraire, Ecole Doctorale de Français Antenne de l'Université Kasdi Merbah- Ouargla,2008-2009.

8- Yassmina Khadra ou la recherche de la vérité, l'Harmattan. Etude de la trilogie sur le malentendu entre l'Occident et l'Orient, Paris,2013.

\section{*Thèses}

1-HALLAL Siham, étude des personnages dans Dounia de Fatima Bakhaï, Université Abderahmane Mira de Bejaia Faculté des lettres et des sciences humaines Ecole Doctorale de Français ,2013

2- Merazga Souheil, L'écrivain de Yasmina Khadra Autobiographie ou Autofiction ? Université Larbi Ben M'Hidi .Oum El Bouaghi * Faculté des Lettres et des Langues, 2014.

3- Karl Ågerup , L'esthétique didactique de Yasmina Khadra Département De Français, Institutionen för franska, italienska och klassiska språk Uppsats för licentiatexamen, Printed in Sweden by US-AB, Stockholm 
2011 Distributor: Department of French, Italian and Classical Languages, Stockholm University

\section{*Sitographie}

1- https://www.jeuneafrique.com/mag/498529/societe/yasmina-khadrales-peuples-du-maghreb-sont-une-meme-nation/

2-https://www.jeuneafrique.com/mag/255175/culture/rentree-litteraireyasmina-khadra-et-boualem-sansal-combat-a-quatre-poings-contre-ladictature/

3-https://www.jeuneafrique.com/133535/culture/les-50-influents-yasminakhadra-crivain.

4- http://festival-ruedeslivres.org/yasmina-khadra/

5- Paris-Match | Publié le 05/05/2019 à 22h04 |Mis à jour le 06/05/2019 à 14h58, Interview Yannick VELY.

6.https://www.google.com.sa/url? sa $=$ t\&rct $=\mathrm{j} \& \mathrm{q}=\&$ esrc $=\mathrm{s} \&$ source $=$ web\&c $\mathrm{d}=25 \& \mathrm{cad}=$ rja\&uact $=8 \& \mathrm{ved}=2$ ahUKEwi59sWBlqznAhUt4KYKHTJy B0I4FBAWMAR6BAgDEAE\&url=https\%3A\%2F\%2Fwww.lavoixdu nord.fr\%2F666413\%2Farticle\%2F2019-11-16\%2Fyasmina-khadraecrivain-algerien-mondialement-connu-valenciennes-lundi-etmardi\&usg=AOvVaw3b_MhNs7hKLNO2cj1m9ZdI

https://www.google.com.sa/url?sa=t\&rct $=\mathrm{j} \& \mathrm{q}=\&$ esrc $=$ s\&source=web\&cd $=26 \& \mathrm{cad}=\mathrm{rja} \&$ uact $=8 \& \mathrm{ved}=2$ ahUKEwi59sWBlqznAhUt4KYKHTJy B0I4FBAWMAV6BAgHEAE\&url=http $\% 3 \mathrm{~A} \% 2 \mathrm{~F} \% 2 \mathrm{Fwww} . r f i . f r \% 2 \mathrm{~F}$ emission\%2F20190628-ecrivain-algerien-yasminakhadra\&usg=AOvVaw3vExNdRM63uD4cf-fA3UL8

8-Youssef Abouali, «La trilogie de Yasmina Khadra. Une œuvre-limite », Sciences humaines combinées [En ligne], 5 |2010, mis en ligne le 11 décembre 2017, consulté le 14 janvier 2020. URL: http://preo.ubourgogne.fr/shc/` index.php?id=171

9- Tristan Mandon "Les Origines de l'Arbre de Mai" dans la cosmogonie runique des Atlantes boréens http://racines.traditions.free.fr

*Vidéo

- You Tube, 13 sept. 2018 - Ajouté par La Grande Librairie Avec « Khalil ", Yasmina Khadra nous plonge dans la tête d'un kamikaze des attentats du 13-Novembre. Une ... 